\title{
Double water compatible molecularly imprinted polymers applied as solid-phase extraction sorbent for selective preconcentration and determination of triazines in complicated water samples
}

\author{
Shoufang $\mathrm{Xu}^{\mathrm{a}}$, Hongzhi Lu ${ }^{\mathrm{a}}$, Lingxin Chen ${ }^{\mathrm{b}, *}$ \\ a School of Chemistry \& Chemical Engineering, Linyi University, Linyi 276005, China \\ ${ }^{\mathrm{b}}$ Key Laboratory of Coastal Zone Environmental Processes and Ecological Remediation, Yantai Institute of Coastal Zone Research, Chinese Academy of \\ Sciences, Yantai 264003, China
}

\section{A R T I C L E I N F O}

\section{Article history:}

Received 19 March 2014

Received in revised form 7 May 2014

Accepted 11 May 2014

Available online 17 May 2014

\section{Keywords:}

Molecularly imprinted polymers

Water-compatible

Triazines

Solid phase extraction

Water sample

\begin{abstract}
A B S T R A C T
In the present work, double water compatible molecularly imprinted polymers (DWC-MIPs) with water compatible core and hydrophilic polymer brushes were prepared by reversible addition-fragmentation chain transfer precipitate polymerization (RAFTPP) and applied as solid-phase extraction (SPE) sorbent for selective preconcentration and specific recognition of triazines in water samples. The DWC-MIPs employed as SPE sorbent presented much higher extraction efficiency for four triazines in aqueous media based on the double water compatible property. The validated method was also successfully applied to tap water and river water sample analysis, and satisfactory recoveries were attained, such as $69.2-95.4 \%$ with the precision of $1.59-3.94 \%$ for four triazines at $100 \mu \mathrm{g} \mathrm{L}^{-1}$. The DWC-MIPs-SPE proves to be a highly effective cleanup and enrichment method for simultaneous separation and sensitive determination of triazines in complicated water samples.
\end{abstract}

(C) 2014 Elsevier B.V. All rights reserved.

\section{Introduction}

Molecular imprinting is known as a technique for creation of tailor-made binding sites with memory of the shape, size and functional groups of the template molecules. Hence, molecularly imprinted polymers (MIPs) are synthetic polymers having a predetermined selectivity for a given analyte, or a group of structurally related compounds $[1,2]$. In the recent years, MIPs have been widely applied and achieved great progress in many fields, such as solid phase extraction (SPE) [3-7] and chemical sensors [8,9], owing to their desired selectivity, physical robustness, thermal stability, as well as low cost and easy preparation.

Up to date, the most widely used technique for preparing MIPs is non-covalent imprinting, in which the complex of template and functional monomer is formed by non-covalent interactions, such as hydrogen bonding, ionic interactions, van der Waals forces, and $\pi-\pi$ interactions. Aprotic and low polar organic solvents are often used for non-covalent imprinting. So, most of produced MIPs can successfully achieve specific recognition in organic solvent-based media. However, they often show poor recognition ability for the

\footnotetext{
* Corresponding author. Tel.: +86 535 2109130; fax: +86 5352109130.

E-mail address: lxchen@yic.ac.cn (L. Chen).
}

target in aqueous environments because the presence of polar solvent can disturb the hydrogen bond formed between template and functional monomer [10]. In addition, the nonspecific hydrophobically binding originated from significant hydrophobic interactions between the MIPs and the template in aqueous media makes the inherent specific recognition ability of MIPs to be obscured [11]. Unfortunately, there are many applications which require MIPs capable of operating in more polar solvents such as methanol, and ultimately water. So, development of water compatible MIPs is urgently desirable.

Initially, two-step extraction method was adopted to solve the problem of water compatibility $[12,13]$. Typically, liquid-liquid extraction was first applied to transfer analyte from aqueous media to organic phase prior to MIPs based extraction. However, these procedures are complicate, time-consuming and analytes may be partly lost in sample preparation steps. So, aqueous-compatible MIPs were highly needed.

For this purpose, different strategies focusing on development of water compatible MIPs (WC-MIPs) were developed. A representative approach was preparing MIPs in water-containing system [14,15]. Row reported [15] that MIPs prepared in methanol-water system showed better molecular recognition ability in aqueous environment than MIPs prepared in organic solvent. The second approach was using hydrophilic 2-hydroxyethyl 
methacrylate (HEMA) [16,17], acrylamide [18] or $\beta$-cyclodextrin [19] as functional monomer in the molecular imprinting process. The third one involved a post-modification procedure for the preformed MIPs. Surface-grafting of hydrophilic polymer brush or chemical bonded hydrophilic functional groups on the surface of the MIP particles were two commonly used methods [20-24]. For example, Zhang's group [22-24] prepared a series of WC-MIPs by grafting of hydrophilic polymer brush, such as poly( $\mathrm{N}$-isopropylacrylamide) (PNIPAM), poly(2-hydrozyethyl methacrylate) (PHEMA) onto the MIP microspheres via surfaceinitiated reversible addition-fragmentation chain transfer (RAFT) polymerization. The introduction of hydrophilic brushes significantly improved their surface hydrophilicity and led to their pure water compatible binding properties.

However, to the best of our knowledge, integrating those three strategies into one system was few reported. In the present work, three kinds of WC-MIPs were prepared using methanol/water as solvent, HEMA as co-functional monomer, PHEMA as hydrophilic polymer brush, respectively. In addition to the simple WC-MIPs, double water compatible MIPs (DWC-MIPs) were also prepared by integrating two or three of them into one system. In this work, we used atrazine, a well-established template, as a model to study synthesis of hydrophilic MIPs. The obtained DWC-MIPs were characterized by scanning electron microscope. The molecular binding selectivity was tested through equilibrium binding analysis. Ultimately, DWC-MIPs were used as SPE sorbent to extract fours kinds of triazines including atrazine, simetryn, propazine and ametryn from water samples. Under optimal conditions, the DWC-MIPs can be successfully applied to preconcentration and separation of triazines from water samples simultaneously.

\section{Experimental}

\subsection{Materials}

Methacrylic acid (MAA), ethyleneglycol dimethacrylate (EGDMA), 2-hydroxyethyl methacrylate (HEMA) were purchased from Sigma-Aldrich (Shanghai, China) and distilled in vacuum prior to use in order to remove stabilizers. 2,2'-Azo-bis-isobutyronitrile (AIBN) were purchased from Shanghai Chemical Reagents Company (Shanghai, China) and recrystallized in methanol prior to use. Atrazine, simetryn, propazine and ametryn were kindly provided by Binzhou Agricultural Technology Co. Ltd. (Shandong, China). Chain transfer agent (CTA) for RAFT polymerization was synthesized as reported [25]. High performance liquid chromatography (HPLC) grade methanol and acetonitrile (ACN) were purchased from Merck (Darmstadt, Germany). Doubly purified deionized water (DDW) was obtained with a Pall Cascada laboratory water system. All other reagents were used as supplied without a further purification step.

\subsection{Preparation of water compatible MIPs (WC-MIPs)}

In the present work, two kinds of WC-MIPs were prepared. One without polymer brushes were prepared by one spot method, and the other with polymer brushes were prepared by two steps method: MIPs particles were prepared firstly, then polymer brushes were grafted to the MIPs particles. The following was the specific procedure.

For preparation of WC-MIPs without polymer brushes, prepolymer solution was prepared by dissolving functional monomer (MAA or HEMA, $2 \mathrm{mmol}$ ) and template (atrazine, $0.5 \mathrm{mmol}$ ) in solvent (methanol or ACN, $60 \mathrm{~mL}$ ), which was stored at $4{ }^{\circ} \mathrm{C}$ in dark for $12 \mathrm{~h}$. Then, cross-linker (EGDMA, $10 \mathrm{mmol}$ ), initiator (AIBN, $20 \mathrm{mg}$ ), and CTA for RAFT living polymerization (CTA, $60 \mu \mathrm{L}$ ) were added to the pre-polymer solution. The solution was degassed by ultrasonic bath for $5 \mathrm{~min}$, and then purged with nitrogen for $10 \mathrm{~min}$. Polymerization was performed in water bath at $60^{\circ} \mathrm{C}$ for $24 \mathrm{~h}$. The resultant polymer particles were washed with methanol/acetic acid solution $(9: 1, v / v)$ to remove both the template molecules and residual monomers. Finally, the particles were dried to constant weight under vacuum at $40^{\circ} \mathrm{C}$.

For preparation of WC-MIPs with polymer brushes, MIPs particles were first prepared as above described, then polymer brushes were grafted to MIPs particles according to the following brief procedure as reported: MIP microspheres with dithioester groups (prepared by above method, $150 \mathrm{mg})$, HEMA $(1.76 \mathrm{~g}, 13.5 \mathrm{mmol})$, CTA ( $7.4 \mathrm{mg}, 27 \mu \mathrm{mol}), \operatorname{AIBN}(1.5 \mathrm{mg}, 9 \mu \mathrm{mol})$ and methanol $(10 \mathrm{~mL})$ were added into a round-bottom flask successively. After being degassed, the grafting polymerization was performed at $70^{\circ} \mathrm{C}$ for $24 \mathrm{~h}$. Then the resulting solid products were thoroughly washed with methanol until no white sediment was detected when ether was added into the washing solutions. For the two kinds of MIPs, non-imprinted polymers (NIPs) were also prepared in the same way but omitting the template in the reaction system.

\subsection{Characterization of the WC-MIPS}

The morphology of the WC-MIPs was evaluated by scanning electron microscope (SEM, Hitachi S-4800, Japan). All samples were sputter-coated with gold before SEM analysis. Molecular recognition properties including rebinding capacity, rebinding kinetics and selectivity experiment were estimated as following: $20 \mathrm{mg}$ WC-MIPs particles were dispersed in $5 \mathrm{~mL}$ flask containing $2.0 \mathrm{~mL}$ atrazine water solutions of various concentrations. After shaking for $24 \mathrm{~h}$ at room temperature, the samples were centrifuged and the supernatant solutions were collected, concentrations of which were determined using HPLC-DAD (Elite, China). The binding amount of atrazine $(Q)$ was determined by subtracting the residual amount of atrazine in solution from the total atrazine amount. $Q$ can be calculated according to the following formula:

$Q=\frac{\left(C_{0}-C_{F}\right) V}{m}$

where $C_{0}\left(\mathrm{mg} \mathrm{mL}^{-1}\right)$ and $C_{\mathrm{F}}\left(\mathrm{mg} \mathrm{mL}^{-1}\right)$ are the initial and final concentration of atrazine solution, respectively. $V(\mathrm{~mL})$ is the sample volume and $m(\mathrm{~g})$ is the mass of the polymer. Meanwhile, the binding kinetics was tested by monitoring the temporal amount of atrazine in the solutions. And the selectivity experiments were carried out by using simetryn, propazine, ametryn, and pentachlorophenol (PCP) as structural analogs. A C18 column with $250 \mathrm{~mm} \times 4.6 \mathrm{~mm}$ i.d. (Waters) was used as the analytical column. HPLC conditions employed for the triazines separation were as follows: mobile phase, acetonitrile:water (40:60, v/v); flow rate, $1.0 \mathrm{~mL} \mathrm{~min}^{-1}$; room temperature; DAD detection, at $222 \mathrm{~nm}$; injection volume, $20 \mu \mathrm{L}$.

\subsection{MIPS-SPE procedures}

A PTFE column was packed with $400 \mathrm{mg}$ WC-MIPs in $5 \mathrm{~mL}$ methanol solution using a wet packing method. Then the cartridge was conditioned with $5 \mathrm{~mL}$ methanol. Atrazine or its structural analogs, dissolved in water, was loaded onto the SPE cartridge at a certain flow rate. Subsequently, the cartridge was washed and eluted. The collected column-solution, washing solution and elute were evaporated to dryness under nitrogen and the residues were redissolved in $0.5 \mathrm{mLACN}$ for HPLC analysis. In order to get the highest SPE recoveries, the variables including the flow rate of loading, loading volume and the elute solvent were studied and optimized. 

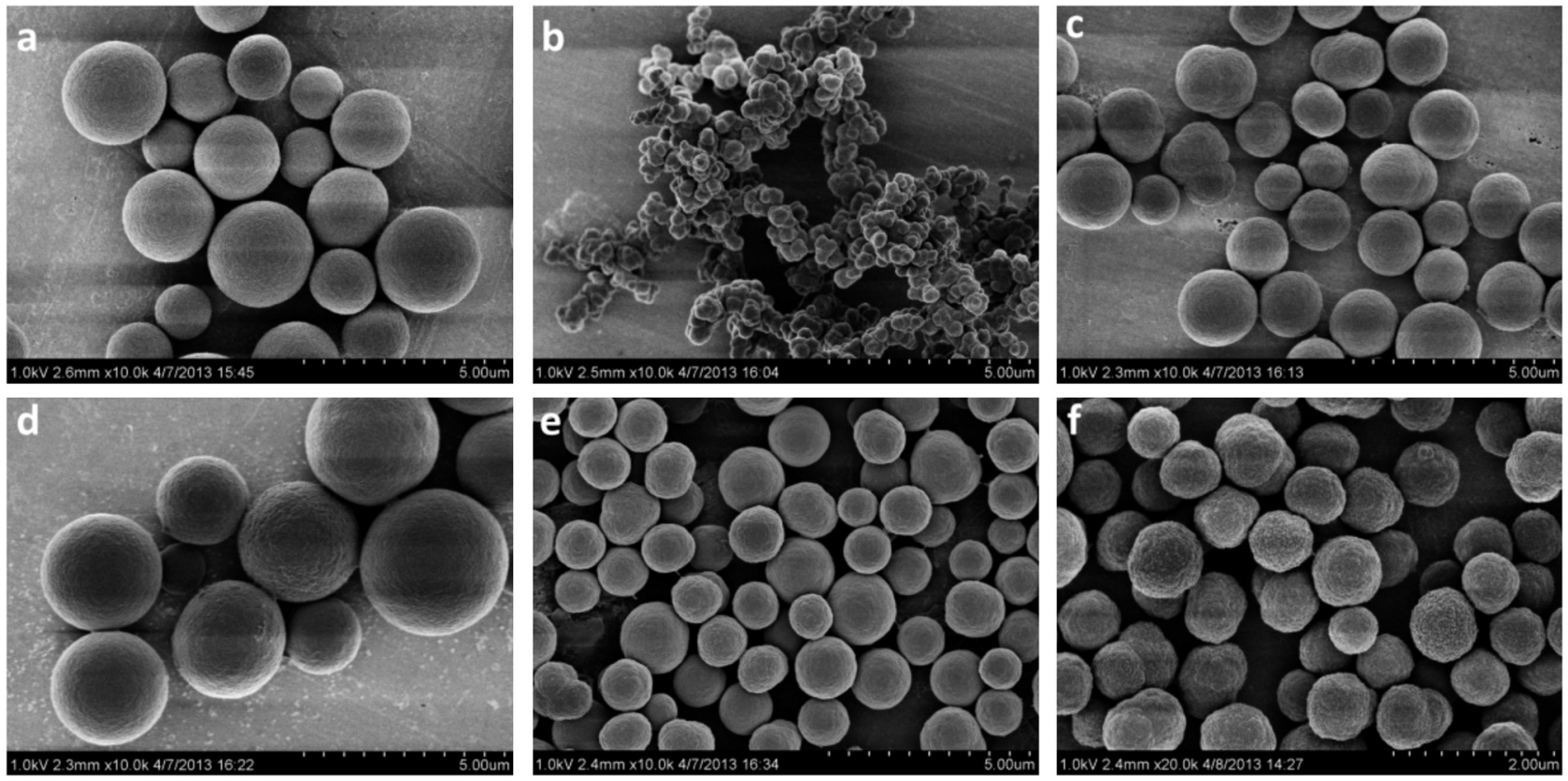

Fig. 1. SEM images of MIPs. (a) MIP-C; (b) MIP1; (c) MIP2; (d) MIP3; (e) MIP4; and (f) NIP for MIP4

\subsection{Analysis of water samples}

Once the optimized MIPs-SPE experimental conditions were established, water samples were used to demonstrate the applicability of the WC-MIPs for preconcentration of triazine herbicides from aqueous matrices. Tap water sample was collected from our laboratory after flowing for about $5 \mathrm{~min}$, and river water sample was collected from Yi River in Linyi city of China. The water samples were collected in Nalgene bottles and filtered through $0.45 \mu \mathrm{m}$ pore size membrane to remove the suspended particles, and then the filtrates were kept in a refrigerator at $4{ }^{\circ} \mathrm{C}$ for use. The water samples were concentrated with the mixture standards of atrazine, ametryn, simetryn, and propazine individual at 50, 100 and $200 \mu \mathrm{g} \mathrm{L}^{-1}$, respectively. The SPE cartridge was conditioned with $5 \mathrm{~mL}$ methanol, and then $100 \mathrm{~mL}$ spiked sample was passed through. Subsequently, the column was washed with $3 \mathrm{~mL}$ DDW, eluted with $5 \mathrm{~mL}$ ethanol, and then the eluted fraction was evaporated to dryness under nitrogen and the residues were redissolved in $0.5 \mathrm{~mL} \mathrm{ACN}$ for HPLC analysis.

\section{Results and discussion}

\subsection{Preparation and characterization of DWC-MIPs}

\subsubsection{Procedures for preparation of WC-MIPS}

Up to date, three kinds of methods have been reported to prepare WC-MIPs. They used methanol or water as solvent, added water soluble functional monomer as co-monomer, and grafted water compatible polymer brushes to MIPs. In the present work, these three kinds of methods were all tried, and the specific recipe was displayed in Table 1 . First, the control MIP (MIP-C) was prepared using MAA as functional monomer, ACN as solvent by precipitate polymerization. MIP-C, with uniform spherical morphology (Fig. 1a), displays well selective recognition ability in organic solvent. Then methanol was used as solvent to prepare MIP1 by precipitate polymerization. The morphology of MIP1 particles was irregular, as seen from Fig. 1b, which was not ideal for molecule recognition. The reasonable explanation was that the polarity and solubility of methanol was not match to the developed polymer. Then co-monomer HEMA was used to improve the water compatibility of MIPs. ACN was the most commonly used solvent for precipitation polymerization, so ACN was still used as solvent. MIP2 particles precipitate from ACN was also uniform spherical (Fig. 1c). For MIP3, MIPs particle was first prepared using MAA as functional monomer and ACN as solvent by RAFT precipitation polymerization (RAFTPP), and then PHEMA was grafted to MIP particles in order to improve the water compatibility. The morphology for MIP3 was also uniform spherical (Fig. 1d). Based on the above results, we can see that MIP2 using HEMA as co-functional monomer was mainly modified the internal structure of MIPs, while MIP3 grafting PHEMA onto the surface of MIPs was changed the "external" of MIPs. In the present work, we integrated these two methods for the synthesis of MIP4. So MIP4 was prepared using HEMA as co-momomer, ACN as solvent, and PHEMA was still grafted to MIP particles by RAFTPP. The procedure was displayed in Fig. 2A. MIP4 has WC-core and WC-shell, so it is also called double WC-MIPs (DWC-MIPs). The morphology of MIP4 was also uniform spherical (Fig. 1e), which can be attributed to the advantage of living polymerization. From Fig. $1 \mathrm{f}$ we can see that DWC-NIPs have similar morphology with DWC-MIPs, so template molecular did not show obvious effect on the morphology of obtained polymers.

The five different kinds of MIPs were also estimated by FT-IR and the results were displayed in Fig. 3. We can see that all five MIPs have the similar peak shape but different peak strength. For the five kinds of MIPs, MIP-C and MIP1 were both polymerized by MAA and EGDMA, MIP2, MIP3 and MIP4 were the copolymer of MAA, HEMA and EGDMA. In addition, MAA and HEMA have the similar functional groups (Fig. 3). Therefore, the FT-IR spectra showed the similar peak shape. However, for MIP3 and MIP4 with PHEMA

Table 1

The specific recipe for preparation WC-MIPs.

\begin{tabular}{llll}
\hline & Monomer & Solvent & Grafting brush \\
\hline MIP-C & MAA & ACN & No \\
MIP1 & MAA & Methanol & No \\
MIP2 & MAA-HEMA & ACN & No \\
MIP3 & MAA & ACN & PHEMA \\
MIP4 & MAA-HEMA & ACN & PHEMA \\
\hline
\end{tabular}



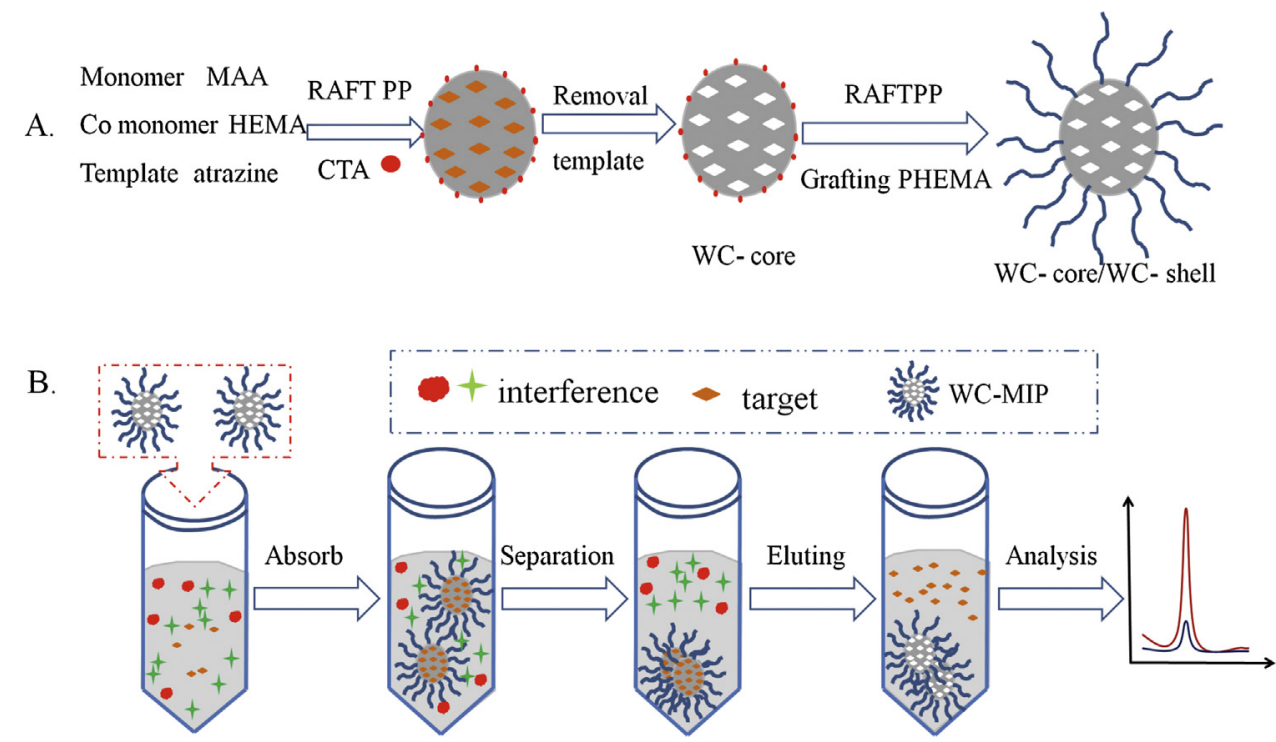

Fig. 2. Schematic illustrations for (A) the procedure for preparation of DWC-MIPs by two steps, and (B) the adsorption behavior of the DWC-MIPs.

polymer brushes, the amount of functional monomers was larger than the other three kinds of MIPs, so they have higher peak strength.

\subsubsection{Estimation of binding properties of the DWC-MIPs}

In addition to the morphology, the binding capacity was an important index to evaluate these methods. Static adsorption experiments were performed to evaluate the adsorption capacities of those WC-MIPs which were dispersed in aqueous solution at concentrations of $0-70 \mathrm{mg} \mathrm{L}^{-1}$ and the results were displayed in Fig. 4A. From Fig. 4A, we can see that MIP-C displayed poor recognition ability in water media. The dominant reason is that MIP-C has low water compatibility, which cannot disperse uniformly in water. For those four kinds of WC-MIPs, MIP1 prepared in methanol has the lowest binding capacity in water, which matches with result of SEM image. MIP2 and MIP3 have better binding capacity in aqueous media because of the improved water compatibility. MIP4, combining the advantage of MIP2 and MIP3, has the highest binding capacity. The result can be explained by Fig. 2B. Due to the presence of hydrophilic polymer brushes, MIP4 have good water compatibility. So polymer particles can uniformly disperse instead of floating

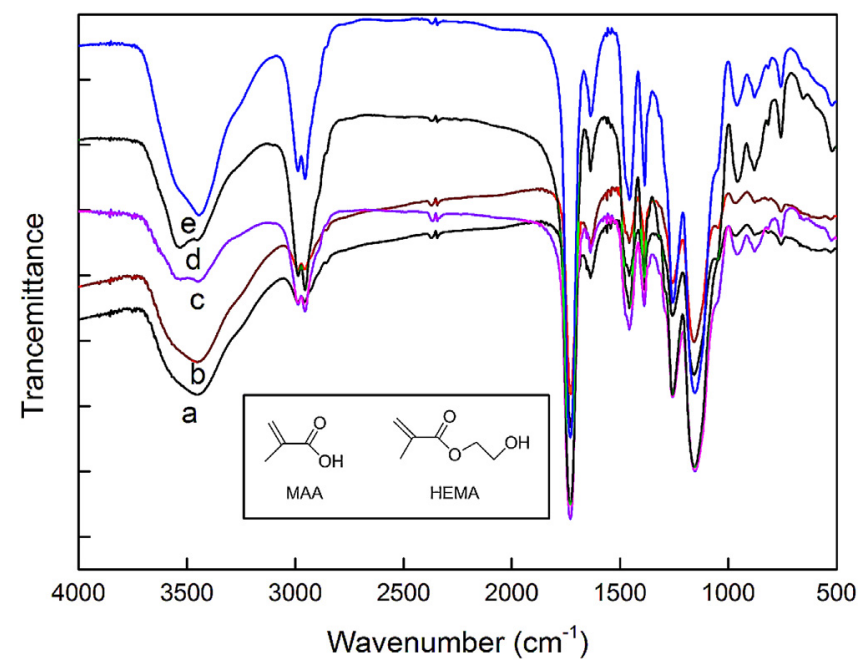

Fig. 3. FT-IR spectra of the WC-MIPs. (a) MIP-C; (b) MIP1; (c) MIP2; (d) MIP3; and (e) MIP4. The inset was the chemical structure of MAA and HEMA. in water, which provides more contact opportunities between polymer particles and analyte. The internal of the polymer particles was full of imprinted sites which have specific selective recognition ability for target molecules. The target molecules in the surface of the polymer can be selected absorbed into those recognition sites, while those interferents still remain in water solution.

In order to determine the binding rate of atrazines on those WC-MIPs, dynamic binding experiments were also carried out. It can be clearly seen from Fig. 4B that the equilibrium time required for four kinds of MIPs was comparable. However, MIP4 had higher adsorption capacity than that of other WC-MIPs within the same time. Moreover, to attain the same adsorption capacity (the equilibrium adsorption capacity of MIP-C), MIP4 needed only one fourth of time required by MIP-C and about half the time required by MIP2 and MIP3. MIP4 had faster template rebinding process than that of other WC-MIPs, which could be attributed to the double water compatibility characteristic of MIP4.

The binding selectivity of MIPs is often determined by comparing the binding amounts of the template with its analogs, which affords an indication of the cross-reactivity of the MIPs toward selected molecules. In the present work, three triazines (simetryn, propazine and ametryn) as structural analogs and one reference PCP (their chemical structures were displayed in Fig. 4D) as other herbicides were used to investigate the competitive recognition ability of WC-MIPs. Fig. 4C displayed that the four kinds of WCMIPs using atrazine as template showed considerable recognition ability for the other three triazines and poor binding capacity for PCP. It also showed that NIPs had similar adsorption capacity for the five compounds. MIPs could recognize template molecule or its structural analogs and it was an important property for enriching a class of target molecules. For the reference PCP, the shape, size and functional groups were different from atrazine, so MIPs prepared using atrazine as template did not show the specific recognition ability. NIPs could also adsorb the five compounds non-selectively because there were no selective recognition sites in NIPs.

\subsection{Analytical performance for the triazines determination based on DWC-MIPS-SPE}

According to the above results, MIP4, the DWC-MIPs, has the highest binding capacity and fastest binding kinetics in aqueous media, so in the following work, DWC-MIPs were employed as SPE 

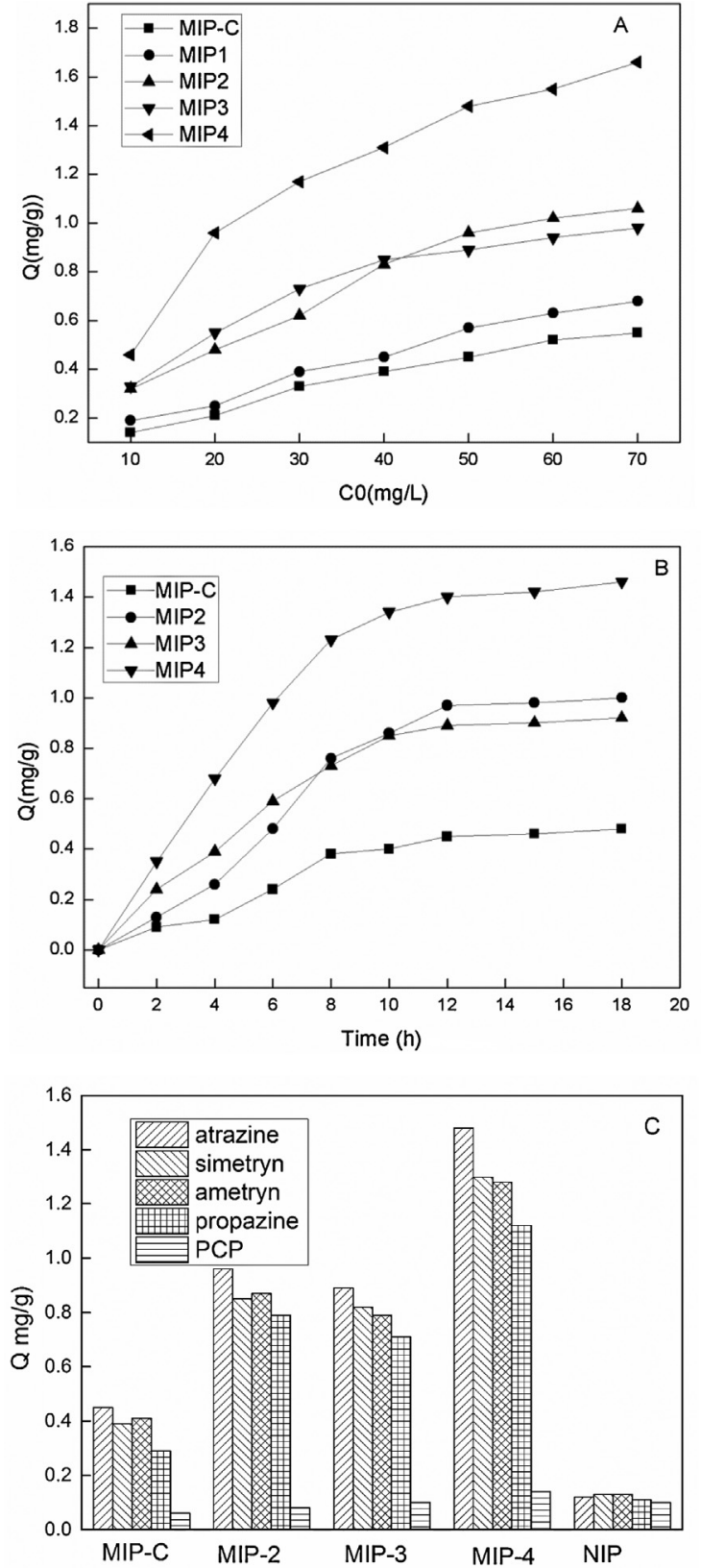<smiles>CCNc1nc(Cl)nc(NC(C)C)n1</smiles>

atrazine

propazine<smiles>CCNc1nc(NC(C)C)nc(SC)n1</smiles>

ametryn<smiles>CCNc1nc(NCC)nc(SC)n1</smiles>

simetryn<smiles>Oc1c(Cl)c(Cl)c(Cl)c(Cl)c1Cl</smiles>

pentachlorophenol
Fig. 4. Binding isotherms of MIP for atrazine in water, which including the static absorb experiment (A), kinetic uptake experiment (B), selective binding experiment $(\mathrm{C})$ and chemical structure for atrazine, ametryn, propazine, simetryn and PCP (D). Experimental conditions for static absorb experiment: $V=2.0 \mathrm{~mL}$; mass of polymer, $20 \mathrm{mg}$; adsorption time, $24 \mathrm{~h}$. Experimental conditions for kinetic uptake experiment: $V=2.0 \mathrm{~mL} ; C_{0}=50 \mathrm{mg} \mathrm{L}^{-1}$; mass of polymer, $20 \mathrm{mg}$. Experimental conditions for selective binding experiment: polymer, $20 \mathrm{mg} ; C_{0}=50 \mathrm{mg} \mathrm{L}^{-1} ; V=2.0 \mathrm{~mL}$; adsorption time, $24 \mathrm{~h}$; room temperature.
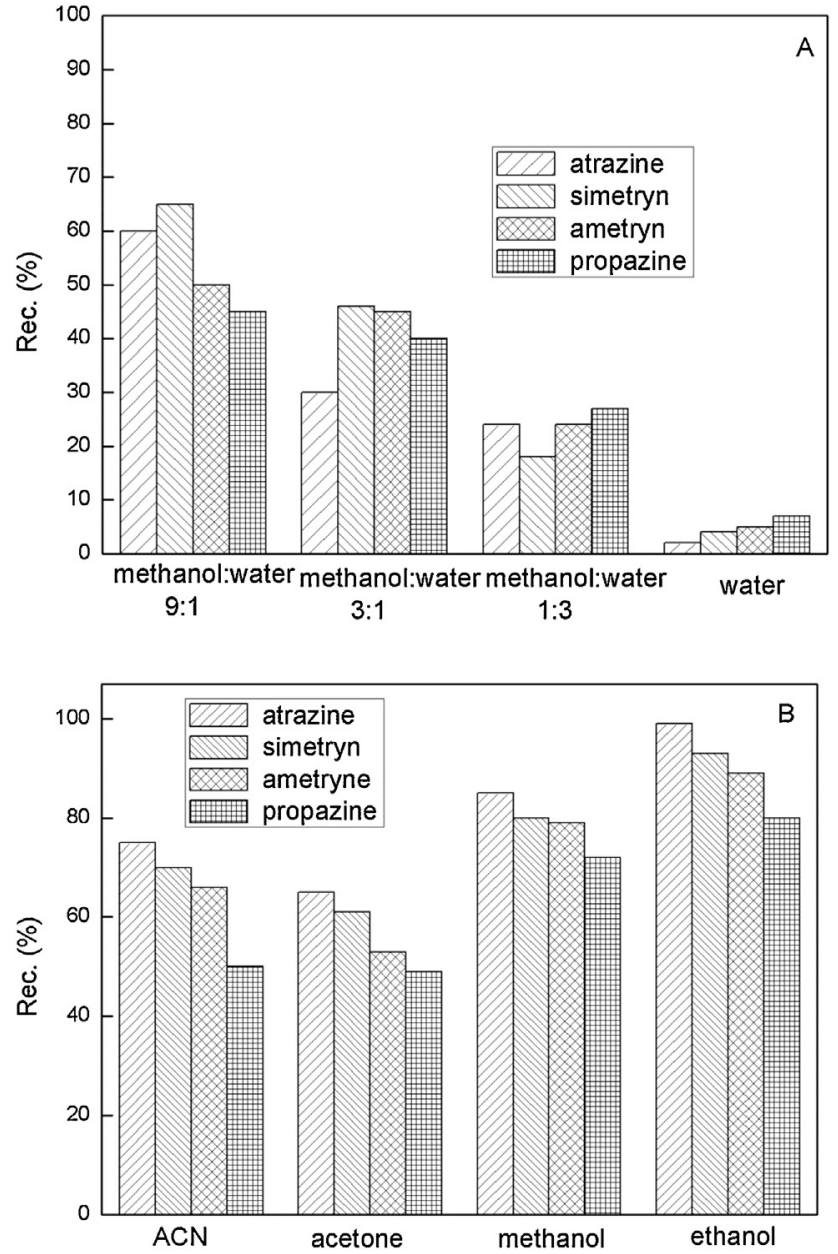

Fig. 5. Effect of the washing solvent (A) and eluting solvent (B) on the recovery of the four triazines.

sorbent for preconcentration of triazines. In order to get the highest extraction recoveries, various influence factors of SPE were studied and optimized, such as the flow rate of loading, loading volume, washing solvent and elution solvent. In the column experiment, $400 \mathrm{mg}$ DWC-MIPs were mixed with methanol and then packed in SPE column. After conditioning with $5 \mathrm{~mL}$ methanol and $5 \mathrm{~mL}$ DDW, $100 \mathrm{~mL}$ of $0.1 \mathrm{mg} \mathrm{L}^{-1}$ atrazine, simetryn, propazine and ametryn mixture solution in water was passed through. The collected column-solution was dried under nitrogen and the residues were redissolved in $0.5 \mathrm{~mL} \mathrm{ACN}$ for HPLC analysis. The flow rate of loading sample was a very important parameter affecting the binding of atrazine. Low loading flow rate would result in a long time of analysis, while high flow rate would decrease the adsorption percentage because equilibrate time was not enough. The flow rate in the range of $0.5-1.5 \mathrm{~mL} \mathrm{~min}^{-1}$ was studied. $100 \%$ of atrazine and $90 \%$ of propazine were bounded to DWC-MIPs when the flow rate was less than $1.0 \mathrm{~mL} \mathrm{~min}^{-1}$. Increasing the loading volume from 50 to $150 \mathrm{~mL}$ at the flow rate of $1.0 \mathrm{~mL} \mathrm{~min}^{-1}, 95 \%$ of atrazine and $85 \%$ of propazine still could be bound to DWC-MIPs when the loading volume was $100 \mathrm{~mL}$. So $1.0 \mathrm{~mL} \mathrm{~min}^{-1}$ and $100 \mathrm{~mL}$ were chosen as the optimal flow rate and loading volume in the following work.

The other important affecting factors for the recoveries of target molecules were the washing solvent and elution solvent. Different ratios of the mixed methanol:water solvents were tested as the washing solvents, $\mathrm{ACN}$, methanol, acetone and ethanol were tested as the eluting solvents. From Fig. $5 \mathrm{~A}$ we can see that when water was used as washing solvent, less than $5 \%$ of the four trazines were found in the washing solvent. The recovery of the four trazines 
Table 2

Linear relations, detection limits and relative standard deviations (RSD, \%, $n=5)$ for the determination of the four triazines.

\begin{tabular}{llllll}
\hline Sample & $\begin{array}{l}\text { Linear range } \\
\left(\mu \mathrm{g} \mathrm{L}^{-1}\right)\end{array}$ & $R$ & $\begin{array}{l}\text { LOD } \\
\left(\mu \mathrm{g} \mathrm{L}^{-1}\right)\end{array}$ & $\begin{array}{l}\text { Interday } \\
\mathrm{RSD}(\%)\end{array}$ & $\begin{array}{l}\text { Intraday } \\
\mathrm{RSD}(\%)\end{array}$ \\
\hline Atrazine & $50-1000$ & 0.9998 & 3.2 & 2.35 & 2.17 \\
Ametryn & $50-1000$ & 0.9992 & 3.8 & 1.97 & 1.84 \\
Simetryn & $50-1000$ & 0.9959 & 4.5 & 3.37 & 3.55 \\
Propazine & $80-1000$ & 0.9877 & 8.6 & 4.25 & 3.98 \\
\hline
\end{tabular}

increased with the increasing of methanol in the washing solvent. So water was used as the washing solvent. For the eluting solvent, $6 \mathrm{~mL}$ ethanol was enough to eluting more than $95 \%$ of triazines, seen from Fig. 5B. So ethanol was used as the eluting solvent in the following work.

Under the optimum experimental conditions, the analytical performances for the present of DWC-MIPs-SPE-HPLC method for the determination of four triazines were evaluated and the results were shown in Table 2 . The linear range for atrazine, ametryn and simetryn was from 50 to $1000 \mu \mathrm{g} \mathrm{L}^{-1}$, while the value was from 80 to $1000 \mu \mathrm{g} \mathrm{L}^{-1}$ for propazine. The calibration curves for the detection of those four triazines were obtained by performing a linear regression analysis. Good linearity was obtained with correlation coefficients of $R>0.98$. The limits of detection (LOD) were $3.2-8.6 \mu \mathrm{g} \mathrm{L}^{-1}$ based on a signal-to-noise ratio of 3. To determine intraday precision, replicate analyses $(n=5)$ at spiked concentration of $100 \mu \mathrm{g} \mathrm{L}^{-1}$ were performed on the same day, and interday precision were determined by repeated the same procedure on different days $(n=5)$ at the same concentration. The precision was given by the relative standard deviations (RSD) of intraday and interday. The intraday repeatability evaluated as RSD was $1.84-3.98 \%$, and the interday reproducibility was $1.97-4.25 \%$. Therefore, the method was demonstrated to be applicable for sensitive and accurate quantitative determination of the triazines in water samples.

\subsection{Applications of DWC-MIPs to water sample analysis}

In order to evaluate the potential applications of DWC-MIPs for selective preconcentration of triazines in real samples, two water sample solutions spiked the mixture standard of atrazine, ametryn, simetryn and propazine individual at 50,100, and $200 \mu \mathrm{g} \mathrm{L}^{-1}$, respectively, were passed through the DWC-MIPs SPE column. Initially, none of those four triazines was detected in the tap water and river water samples. After spiking, four compounds can be remarkably concentrated by the MIPs-SPE (Fig. 6). Comparing DWC-MIPs-SPE (Fig. 6a) with MIP-C-SPE (Fig. 6b), DWC-MIPs displayed higher preconcentration ability. The results can be attributed to the fact that DWC-MIPs have much higher imprinting efficiency in aqueous media. For the NIPs-SPE, no obvious peaks of triazines were observed at the corresponding retention time
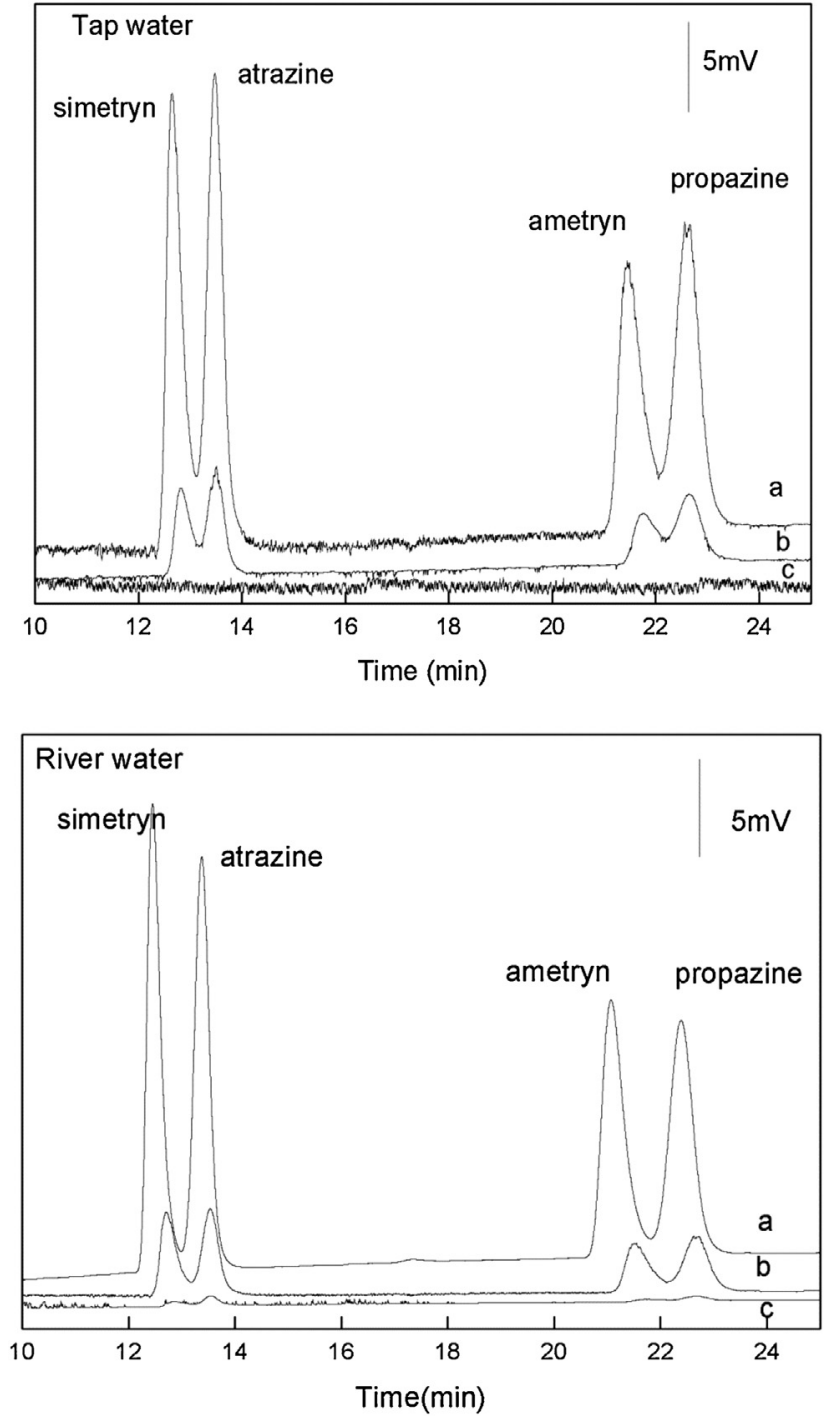

Fig. 6. HPLC-DAD chromatograms of $100 \mu \mathrm{g} \mathrm{L}^{-1}$ triazines mixture standard spiked water samples: (a) extraction with DWC-MIPs, (b) extraction with MIP-C, and (c) extraction with NIP. (1) simetryn, (2) atrazine, (3) ametryn and (4) propazine. Experimental conditions: $100 \mathrm{~mL}$ spiked solutions; $400 \mathrm{mg}$ DWC-MIPs; wash solution, $5 \mathrm{~mL}$ water; elution solution, $5 \mathrm{~mL}$ ethanol; re-dissolution solution, $500 \mu \mathrm{L}$ ACN. HPLC condition: mobile phase, acetonitrile: water $(40: 60, v / v)$; flow rate, $1.0 \mathrm{~mL} \mathrm{~min}^{-1}$; room temperature; DAD detection, $\lambda 220 \mathrm{~nm}$; injection volume, $20 \mu \mathrm{L}$.

(Fig. 6c), indicating the triazines were specifically adsorbed on the MIPs but negligible interaction on the NIPs.

The validation of the DWC-MIPs-SPE method was performed by examining the recoveries of spiked water samples. The precision of the method was evaluated by calculating the RSD of the

Table 3

MIP4-SPE recoveries (Rec.) and relative standard deviations (RSD, \%) obtained from analysis of tap water and river water samples spiked with four kinds of triazines.

\begin{tabular}{|c|c|c|c|c|c|c|c|}
\hline \multirow[t]{2}{*}{ Sample } & \multirow[t]{2}{*}{ Triazines } & \multicolumn{2}{|c|}{$50 \mu \mathrm{g} \mathrm{L}^{-1}$} & \multicolumn{2}{|c|}{$100 \mu \mathrm{g} \mathrm{L}^{-1}$} & \multicolumn{2}{|c|}{$200 \mu \mathrm{g} \mathrm{L}^{-1}$} \\
\hline & & Rec. (\%) & RSD (\%) & Rec. (\%) & RSD (\%) & Rec. (\%) & $\operatorname{RSD}(\%)$ \\
\hline \multirow[t]{4}{*}{ Tap water } & Atrazine & 83.3 & 1.58 & 95.4 & 2.12 & 97.6 & 3.56 \\
\hline & Ametryn & 80.7 & 2.37 & 88.7 & 2.54 & 90.5 & 2.57 \\
\hline & Simetryn & 81.2 & 1.98 & 86.4 & 3.13 & 93.2 & 2.98 \\
\hline & Propazine & 66.4 & 3.15 & 70.2 & 3.86 & 85.2 & 4.05 \\
\hline \multirow[t]{4}{*}{ River water } & Atrazine & 88.2 & 1.33 & 92.7 & 1.59 & 94.7 & 2.58 \\
\hline & Ametryn & 86.7 & 2.28 & 89.6 & 2.13 & 93.8 & 3.07 \\
\hline & Simetryn & 76.4 & 3.06 & 79.8 & 2.78 & 89.5 & 3.12 \\
\hline & Propazine & 65.3 & 4.73 & 69.2 & 3.94 & 83.3 & 4.17 \\
\hline
\end{tabular}


extraction at different concentration levels using the optimized procedures. The results are listed in Table 3. Satisfactory recoveries were obtained, such as $70.2-95.4 \%$ with precision of $2.12-3.86 \%$ at $100 \mu \mathrm{g} \mathrm{L}^{-1}$ for tap water sample, $69.2-92.7 \%$ with precision of $1.59-3.94 \%$ for river water sample. This demonstrated the potential applicability of the DWC-MIPs for simultaneous preconcentration, separation, sensitive determination and accurate quantification of triazines in water samples.

\section{Conclusions}

In this present work, four types of atrazine imprinted WC-MIPs, including MIP1, using methanol as solvent, MIP2, using HEMA as comonomer, MIP3, grafting PHEMA to MIP particles, and MIP4 with water compatible MIP core and hydrophilic PHEMA brushes were successfully prepared. Among those four kinds of WC-MIPs, DWCMIPs displayed excellent recognition ability in aqueous media owing to the double water compatible characteristic: they have water compatible core and water compatible polymer brush simultaneously. The SPE based on DWC-MIPs was successfully used for simultaneous preconcentration, separation and determination of four triazine compounds in tap water and river water samples. Given the high specificity and high enrichment ability of DWCMIPs, we expect that they can be applied for trace pollutes detection in complicated water matrices. In addition, selective removal and catalytic photodegradation of the target in water is also a very important objective. So, preparing WC-MIPs with photodegradation ability are currently in progress.

\section{Acknowledgements}

Financial support from the National Natural Science Foundation of China (21307052 and 21175158), the Natural Science Foundation of Shandong Province of China (ZR2013BL006), and the Scholarship Award for Excellent Doctoral Student granted by the Ministry of Education of China, is gratefully acknowledged.

\section{References}

[1] L.J. Pauling, J. Am. Chem. Soc. 62 (1940) 2643.

[2] G. Vlatakis, L.l. Andersson, K. Mosbach, Nature 361 (1993) 645.

[3] S.F. Xu, J.H. Li, L.X. Chen, J. Mater. Chem. 21 (2011) 4346.

[4] S.F. Xu, L.X. Chen, J.H. Li, W. Qin, J.P. Ma, J. Mater. Chem. 21 (2011) 12047.

[5] S.F. Xu, L.X. Chen, J.H. Li, Y.F. Guan, H.Z. Lu, J. Hazard. Mater. 237-238 (2012) 237.

[6] S.F. Xu, J.H. Li, X.L. Song, J.S. Liu, H.Z. Lu, L.X. Chen, Anal. Methods 5 (2013) 124

[7] Z. Zhang, S.F. Xu, J.H. Li, H. Xiong, H.L. Peng, L.X. Chen, J. Agric. Food Chem. 60 (2012) 180.

[8] M.J. Whitcombe, I. Chianella, L. Larcombe, S.A. Piletsky, J. Novle, R. Porter, A. Horgan, Chem. Soc. Rev. 40 (2011) 1547.

[9] G. Guan, B. Liu, Z. Wang, Z. Zhan, Sensor 8 (2008) 8291.

[10] L.X. Chen, S.F. Xu, J.H. Li, Chem. Soc. Rev. 40 (2011) 2922.

[11] B. Dirion, Z. Cobb, E. Schilinger, L.I. Andersson, B. Sellergren, J. Am. Chem. Soc. 125 (2003) 15101

[12] X.G. Hu, J.L. Pan, Y.L. Hu, Y. Huo, G.K. Li, J. Chromatogr. A 1188 (2008) 97.

[13] Y.L. Hu, R.J. Liu, Y. Zhang, G.K. Li, Talanta 79 (2009) 579.

[14] H.W. Sun, F.X. Qiao, J. Chromatog. A 1212 (2008) 1.

[15] H.Y. Yan, K.H. Row, G.L. Yang, Talanta 75 (2008) 227.

[16] H. Yan, M. Tian, K.H. Row, J. Sep. Sci. 31 (2008) 3015

[17] E. Benito-Peña, S. Martins, G. Orellana, M.C. Moreno-Bondi, Anal. Bioanal. Chem. 393 (2009) 235.

[18] Z. Meng, W. Chen, A. Mulchandani, Environ. Sci. Technol. 39 (2005) 8958.

[19] L. Qin, X.W. He, W.Y. Li, Y.K. Zhang, J. Chromatog. A 1187 (2008) 94.

[20] K.G. Yang, M.M. Berg, C.S. Zhao, L. Ye, Macromolecules 42 (2009) 8739.

[21] F. Puoci, F. Iemma, G. Cirillo, M. Curcio, O.E. Parisi, U.F. Spizzirri, N. Picci, Eur. Polym. J. 45 (2009) 1634

[22] G.Q. Pan, Y. Ma, Y. Zhang, X.Z. Guo, C.X. Li, H.Q. Zhang, Soft Matter 7 (2011) 8428.

[23] G.Q. Pan, Y. Zhang, Y. Ma, C.X. Li, H.Q. Zhang, Angew. Chem. Int. Ed. 50 (2011) 11731.

[24] Y. Ma, Y. Zhang, M. Zhao, X.Z. Guo, H.Q. Zhang, Chem. Commun. 48 (2012) 6217.

[25] S.F. Xu, J.H. Li, L.X. Chen, Talanta 85 (2011) 282. 